\title{
IL-37 induces anti-tumor immunity by indirectly promoting dendritic cell recruitment and activation in hepatocellular carcinoma
}

This article was published in the following Dove Press journal: Cancer Management and Research

\author{
Yuan Liu ${ }^{1,2, *}$ \\ Jing-Jing Zhao ${ }^{1,2, *}$ \\ Zi-Qi Zhou ${ }^{1,2}$ \\ Qiu-Zhong Pan ${ }^{1,2}$ \\ Qian Zhu ${ }^{1,2}$ \\ Yan Tang ${ }^{1,2}$ \\ Jian-Chuan Xia ${ }^{1,2}$ \\ De-Sheng Weng ${ }^{1,2}$
}

'State Key Laboratory of Oncology in South China, Collaborative Innovation

Center for Cancer Medicine, Sun Yat-Sen

University Cancer Center, Guangzhou,

People's Republic of China; ${ }^{2}$ Department

of Biotherapy, Sun Yat-Sen University

Cancer Center, Guangzhou, People's

Republic of China

*These authors contributed equally to this work
Correspondence: De-Sheng Weng; Jian-Chuan Xia

State Key Laboratory of Oncology in South China, Department of Biotherapy, Sun Yat-sen University Cancer Center, 65I Dongfeng Road East, Guangzhou 510060 , People's Republic of China Tel +862087345699

Fax +86 2087343392

Email wengdsh@sysucc.org.cn;

xiajch@mail.sysu.edu.cn
Introduction: IL-37 is a cytokine of IL-1 family that plays an important role in innate immunity and inflammation, and has been studied as a tumor suppressor in many cancers. However, it remains unclear whether IL-37 plays a regulatory role in tumor-infiltrating dendritic cells (DCs) in hepatocellular carcinoma (HCC).

Materials and methods: We evaluated the relationship between IL-37 expression and tumor infiltration by DCs in 155 HCC samples through immunohistochemical analysis and Kaplan-Meier survival analysis. The effects of IL-37 on the anti-tumor activity of DCs were investigated by ELISA, flow cytometry, real-time quantitative PCR, cytotoxicity assays and tumorigenicity assays.

Results: The expression level of IL-37 in HCC samples was positively correlated with the degree of $\mathrm{CD} \mathrm{a}^{+}$DCs infiltration. The survival rates of patients with both a high expression of IL-37 and a high infiltration by CD1a ${ }^{+}$DCs were significantly higher than those of patients with a low expression of IL-37 and a low infiltration by CD1a ${ }^{+}$DCs. In vitro chemotaxis analysis indicated that HCC cells overexpressing IL-37 recruited more DCs by secreting higher levels of specific chemokines (eg, CCL3 and CCL20). In addition, IL-37 indirectly up-regulated the expression of major histocompatibility class II molecules, CD86 and CD40 on DCs by acting on tumor cells; IL-37 also indirectly enhanced the anti-tumor effect of T lymphocytes by stimulating DCs to secrete cytokines such as IL-2, IL-12, IL12 p 70 , interferon- $\alpha$ (IFN- $\alpha$ ) and IFN- $\gamma$. Finally, overexpression IL-37 in HCC cells significantly delayed tumor growth and increased recruitment of CD11 $\mathrm{c}^{+} \mathrm{DCs}$ to tumor tissues was also revealed in vivo mouse model.

Conclusion: DCs play an important role in IL-37 mediated anti-tumor immune responses in HCC, which may contribute to the development of novel cancer immunotherapeutic strategies.

Keywords: IL-37, dendritic cells, antitumor immunity, hepatocellular carcinoma

\section{Introduction}

Hepatocellular carcinoma (HCC) is the sixth most common malignancy worldwide and is a leading cause of cancer-related mortality. ${ }^{1}$ Although the effectiveness of early diagnosis and treatment has been significantly improved by the implementation of surveillance programs and individualized treatment in recent years, the HCC incidence of morbidity and mortality is still on the rise. ${ }^{2}$ The current effective treatments are limited to the following methods: surgical resection, ablation, and

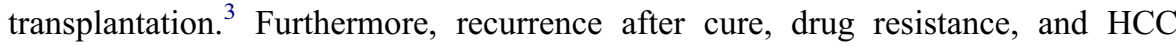
heterogeneity are all difficult problems that are yet to be solved. ${ }^{4,5}$ Therefore, 
exploring new treatment strategies and improving current treatment methods are both urgently needed to solve this global public health problem.

Recent work has revealed the mechanisms underlying tumor immunosuppression and immune escape. Dendritic cells (DCs), as initiators of the naive $\mathrm{T}$ cell response, are key regulators of immune tolerance and activation, are essential for inducing and maintaining anti-tumor immune responses. Moreover, DCs are considered to be the most effective professional antigen-presenting cells (APCs). In the context of tumor immune escape, cancer vaccination, and the anti-tumor immune response, DCs have been extensively studied. ${ }^{6,7}$ Decreased expression levels of major histocompatibility class I (MHC I) molecules and co-stimulatory molecules present on the DCs surface are significant contributors to tumor immune escape. ${ }^{8}$ DCs have also recently been reported to play important roles in both the development and immunosuppression of HCC. ${ }^{9}$ A subset of CD $14^{+}$CTLA- $4^{+}$regulatory dendritic cells $\left(\mathrm{CD} 14^{+} \mathrm{DCs}\right)$ have been identified that inhibit the anti-tumor immune response through the CTLA-4-dependent production of IL-10 and indoleamine 2,3-dioxygenase in HCC. ${ }^{10}$ Patients with HCC also exhibit diminished functioning of DCs in their peripheral blood and fewer mature and activated DCs in their liver tissue. ${ }^{11}$ Importantly, a functional defect in DCs leads to impaired stimulation of allogeneic T cells. ${ }^{11,12}$

IL-37, a member of the IL-1 family, is essential to the inhibition of innate immunity and inflammation and plays a role in the inhibition of cytokines, chemokines, and neutrophil infiltration. ${ }^{13}$ IL-37 has also been reported to have antitumor effects in cancers such as colon cancer, cervical cancer, and prostate cancer. ${ }^{14-16}$ The mechanism of tumor suppression by IL-37 is dependent on cancer type. For instance, IL-37 inhibits the STAT4 signaling pathway in cervical and renal cancer. ${ }^{17}$ The anti-tumor effect of IL-37 in breast cancer is $\mathrm{CD} 4^{+} \mathrm{T}$ cell-dependent. ${ }^{18}$ We previously found that the IL-37 expression level is lower in tumor tissue, compared with adjacent tissue and negatively correlates with tumor size in HCC. However, age, gender, histological differentiation, liver cirrhosis, HBV, serum AFP, recurrence and distant metastasis had no correlates with IL-37 expression. Patients with high expression level of IL-37 in tumor tissue have better prognosis, including overall survival (OS) and disease-free survival (DFS), and the anti-tumor effect of IL-37 in HCC reflects $\mathrm{CD} 57^{+}$natural killer (NK) cell dependence. ${ }^{19}$ However, the role of IL-37 in modulating other tumor-infiltrating immune cells in the tumor microenvironment of HCC remains unclear. Therefore, we analyzed the relationship between IL-37 expression and DCs infiltration in patients with HCC and their effect on prognosis, then investigated the specific effects of IL-37 on the function of DCs.

\section{Materials and methods}

\section{Patients and tissue specimens}

Paraffin-embedded tissues and clinical data were obtained from the Sun Yat-sen University Cancer Center. The total number of patients included in this study was 155 . Written informed consent for each patient was obtained, which was conducted in accordance with the Declaration of Helsinki. All of the patients had not received any treatment before surgery and were followed up for $>5$ years. The period of time from surgery to recurrence or the last follow-up without recurrence was defined as DFS. The period of time from surgery to death or the last known follow-up was defined as OS. This study was approved by the Ethics Committee of the Sun Yat-sen University Cancer Center.

\section{Immunohistochemical analysis}

Paraffin-embedded tissue sections $(2 \mu \mathrm{m})$ collected from HCC patients and animal experiments were used for immunohistochemistry. All sections were heated in the oven for 1 $\mathrm{hr}$ at $65^{\circ} \mathrm{C}$ and rehydrated using graded ethanol. In order to adequately expose antigen, slides were boiled in EDTA (1 $\mathrm{mmol} / \mathrm{L}, \mathrm{pH}$ 8.0) for $15 \mathrm{mins}$ in a microwave oven and naturally cooled to room temperature. To block endogenous peroxidase activity, the sections were washed for twice with PBS soaked in 3\% hydrogen peroxide for 15 mins at room temperature. Then, all slides from HCC patients were incubated with primary monoclonal antibodies: mouse antihuman IL-37 (1:1,000 dilution; Abcam, Cambridge, UK), mouse anti-human CD1a (1:100 dilution; Zhongshan Golden Bridge Biotech Co., Ltd, Beijing, China). All slides from animal experiments were incubated with primary monoclonal antibodies: rabbit anti-mouse IL-37 (1:1,000 dilution; Tianjun, Guangzhou, China), rabbit anti-mouse CD11c (1:2,000 dilution; Tianjun), rabbit anti-mouse CD4 (1:1,000 dilution; Tianjun) and rabbit anti-mouse CD8 (1:1,000 dilution; Tianjun) at $4^{\circ} \mathrm{C}$ overnight. All slides were washed with PBS three times again and incubated with horseradish peroxidase-conjugated secondary antibody (Envision Detection Kit, Dako, Copenhagen, Denmark) for 30 mins at room temperature, then washed with PBS three 
times. Finally, sections were treated with 3,3'-diaminobenzidine tetrahydrochloride and observed with a microscope, counterstained with $20 \%$ hematoxylin, dehydrated, cleared, and evaluated.

\section{Image quantification}

Total IL-37 and CD1a immunostaining was determined by scoring the percentage of positively stained tumor cells and the intensity of staining. Percent positive scores were determined to be " 0 " ( $<5 \%$, negative), " 1 " (5-25\%, sporadic), " 2 " (25-50\%, focal), or " 3 " ( $>50 \%$, diffuse). Staining intensity scores were determined to be " 0 " (negative), " 1 " (faint staining), "2" (moderate staining), or " 3 " (strongly staining). The result of multiplying percent positive score and staining intensity score was used to calculate the total IL-37 and CD1a immunostaining score and ranged from 0 to 9 . The expression level of IL-37 protein and the infiltration by $\mathrm{CD} \mathrm{a}^{+}$DCs was classified as high (final immunostaining score fell within the range of 4-9) or low (final immunostaining score fell within the range of $0-3)$. The densities of CD1a, CD11c, CD4 and CD8 positively stained cells were manually counted under high-power $(400 \times)$ magnification in five randomly selected fields and then averaged (cells/HPF).

\section{Cell lines and culture}

The human HCC cell line Hep3B and mouse HCC cell line Hepa 1-6 (syngeneic to $\mathrm{C} 57 \mathrm{BL} / 6$ mice) were obtained from the American Type Culture Collection (Manassas, VA, USA). Hep3B cells were cultured in DMEM medium (Thermo Fisher Scientific, Waltham, MA, USA) supplemented with 10\% heat-inactivated FBS (Thermo Fisher Scientific) and $1 \%$ penicillin-streptomycin. Hepa 1-6 cells were cultured in RPMI 1640 medium (Thermo Fisher Scientific) supplemented with $10 \%$ heat-inactivated FBS and $1 \%$ penicillin-streptomycin. All cells were incubated at $37^{\circ} \mathrm{C}$ in a humidified chamber containing $5 \% \mathrm{CO}_{2}$.

\section{Overexpression of IL-37 in HCC cells}

Hep3B and Hepa $1-6$ cells $\left(8 \times 10^{5} / 2\right.$ mL DMEM medium or RPMI 1640 medium per well) were plated in six-well culture dishes and incubated for $24 \mathrm{hrs}$. Then, in the presence of $5 \mu \mathrm{g} / \mathrm{mL}$ polybrene (Sigma-Aldrich, St. Louis, MO, USA), the cells were infected with the lentiviruses (LV-NC and LV-IL37) at a multiplicity of infection of 40 . Puromycin $(5 \mu \mathrm{g} / \mathrm{mL})$ was used to select stable cell lines (Hep3B/LV-NC and Hep3B/LV-IL37; Hepa 1-
6/LV-NC and Hepa 1-6/LV-IL37), and the successful induction was confirmed by using Western blotting and ELISA.

\section{Immune cell isolation and culture}

Human peripheral blood mononuclear cells (PBMCs) were isolated from buffy coats (Guangzhou Blood Center, Guangzhou, China) by Ficoll-Hypaque density gradient centrifugation. PBMCs were then seeded $\left(1 \times 10^{8}\right.$ cells $)$ into $75 \mathrm{~cm}^{2}$ culture bottles for $2 \mathrm{hrs}$. Nonadherent cells were collected and cultured in Quanta-007 lymphocyte medium (PAA, Freiburg, Germany) supplemented with IL-2 $(20 \mathrm{U} / \mathrm{ml})$ for $\mathrm{T}$ cell isolation. Dynabeads magnetic beads (Life Technologies, Shanghai, China) were used to isolate $\mathrm{CD}^{+}{ }^{+} \mathrm{T}$ lymphocytes by negative selection according to the manufacturer's instructions. Culture bottles were washed twice with PBS, unwashed cells were treated with $400 \mathrm{U} / \mathrm{mL}$ IL-4 (Thermo Fisher Scientific) and 1,000 U/mL GMCSF (Thermo Fisher Scientific) for 5 days. Then, suspended cells were collected and treated with $10 \mu \mathrm{g} / \mathrm{mL}$ OK-432 (Shandong Lukang Pharmaceutical Co., Ltd, Jining, China) for $24 \mathrm{hrs}$ to generate mature DCs (mDCs).

\section{DCs loaded with antigen of Hep3B cells}

The cryopreserved Hep3B cells in liquid nitrogen tank were placed in warm water $\left(37^{\circ} \mathrm{C}\right)$. After dissolved, the cells were put into liquid nitrogen tank again for rapid freezing, repeated four times, and the supernatant was retained after centrifugation. Then, cell lysates of Hep3B cells were obtained. The mDCs were cultured in medium containing Hep3B cell lysates for $24 \mathrm{hrs}$.

\section{Chemotaxis assays in vitro}

Hep3B/LV-IL37 and Hep3B/LV-NC cells $\left(2 \times 10^{5} / 2 \mathrm{ml}\right.$ DMEM per well) were plated in six-well culture dishes and incubated for $72 \mathrm{hrs}$. Then, culture supernatants were collected and stored at $-80^{\circ} \mathrm{C}$ as aliquots. Polycarbonate filters with a 5 - $\mu \mathrm{m}$ pore size in 24-well transwell chambers (Corning Inc, Corning, NY, USA) were used to perform chemotaxis assays. Purified mDCs $\left(5 \times 10^{6}\right.$ cells $)$ suspended in $100 \mu \mathrm{L}$ of complete medium were placed in the upper chamber, and $600 \mu \mathrm{L}$ of one of the following was added to the lower chamber: supernatant from a Hep3B/LV-IL37 cell culture, supernatant from a Hep3B/ LV-NC cell culture, DMEM containing recombinant IL-37 (the content of IL-37 in DMEM was similar to 
that in the supernatant of Hep3B/LV-IL37 cells), or DMEM alone. Each experiment was performed in triplicate. Cells were incubated for $4 \mathrm{hrs}$ at $37^{\circ} \mathrm{C}$. Then, cells migrated to the bottom chamber were collected, suspended and counted.

\section{Real-time quantitative PCR ( $q P C R$ )}

The TRIzol reagent (Invitrogen, Carlsbad, CA, USA) was used to extract total RNA from the Hep3B/LV-IL37 cells, Hep3B/LV-NC cells, and DCs (cultured in DMEM with or without recombinant IL-37, Hep3B/LV-NC cell culture supernatant, Hep3B/LV-IL37 cell culture supernatant). The SYBR Green Master Mix (Invitrogen) was used to evaluate the expression levels of mRNAs encoding CCL chemokines, CXC chemokines, and GAPDH in an ABI Prism 7900HT Sequence Detection System (Life Technologies, Carlsbad, CA, USA). The specific primers used are presented in Table S1. Data were calculated and analyzed using the comparative threshold cycle $\left(2^{-\Delta \Delta \mathrm{Ct}}\right)$ method.

\section{Flow cytometry analysis}

Mature DCs (mDCs) loaded with antigen of Hep3B cells (Hep3B cell lysates) were cultured for $24 \mathrm{hrs}$ in DMEM alone, with recombinant IL-37, with Hep3B/LV-NC cell culture supernatant, or with Hep3B/LV-IL37 cell culture supernatant, two duplicate wells. Half of the mDCs were used to detect the expression level of CD80, CD86, CD40, MHC Class I and MHC Class II. Half of the mDCs were incubated with purified $\mathrm{CD}^{+} \mathrm{T}$ cells to detect interferon- $\gamma$ (IFN- $\gamma$ ) expression of cytotoxic $\mathrm{T}$ lymphocytes (CTLs) and the proportion of IFN- $\gamma^{+} \mathrm{CD} 8^{+} \mathrm{T}$ cells. The antibodies applied in the assay are as follows: FITC-conjugated antibodies: anti-MHC Class I, anti-MHC Class II, anti-CD80, anti-CD86, anti-CD40 and anti-CD8; APC-conjugated anti-IFN- $\gamma$ (all from BD Biosciences, Franklin Lakes, NJ, USA). The data analysis of CD80, CD86, CD40, MHC Class I, MHC Class II and CD8 was performed using a Cytomics FC 500 Flow Cytometer (Beckman Coulter, Brea, CA, USA). The data analysis of IFN- $\gamma$ was performed using the CXP Analysis software (Beckman Coulter).

\section{Lymphocyte proliferation assay}

The CellTrace CFSE Cell Proliferation Kit (Molecular Probes, Shanghai, China) was used to determine the number of active $\mathrm{T}$ cells according to the manufacturer's protocol.

\section{Generation of CTLs and cytotoxicity}

\section{assays in vitro}

Mature DCs (mDCs), loaded with antigen of Hep3B cells (Hep3B cell lysates), were cultured for $24 \mathrm{hrs}$ in DMEM alone, with recombinant IL-37, with Hep3B/LV-NC cell culture supernatant, or with Hep3B/LV-IL37 cell culture supernatant; the cells were then collected, washed extensively, and incubated with purified $\mathrm{CD}^{+} \mathrm{T}$ cells for 7 days to generate CTLs. Then, CTLs were co-cultured with Hep3B cells at a ratio of $1: 1,3: 1$, or 10:1 for $12 \mathrm{hrs}$. The cytotoxicity of CTLs was assessed using a CytoTox 96 Lactate Dehydrogenase Assay Kit (Promega, Madison, WI, USA) according to the manufacturer's protocol.

\section{ELISA}

Mature DCs (mDCs), loaded with antigen of Hep3B cells (Hep3B cell lysates), were cultured in DMEM alone, with recombinant IL-37, with Hep3B/LV-NC cell culture supernatant, or with Hep3B/LV-IL37 cell culture supernatant. A commercially available ELISA Kit (4A Biotech Co, Ltd, Beijing, China) was used to examine the level of CXCL10, IL-2, IL-6, IL-10, IL-12, IL-12p70, TNF- $\alpha$, IFN- $\alpha$ and IFN- $\gamma$ secreted into the supernatants by mDCs according to the manufacturer's protocol. The level of IL-37 secreted by Hep3B/LV-IL37 cells, Hep3B/LV-NC cells, Hepa 1-6/LVIL37 cells or Hepa 1-6/LV-NC cells was similarly examined.

\section{HCC proliferation assays}

Hepa 1-6/LV-IL37 cells and Hepa 1-6/LV-NC cells were counted and seeded in 96-well plates at 1,000 cells/200 $\mu \mathrm{L}$ per well. The MTS cell proliferation kit (Promega) was used to measure the proliferation rates of Hepa $1-6 / \mathrm{LV}$ IL37 cells and Hepa 1-6/LV-NC cells according to the manufacturer's instructions.

\section{Tumorigenicity assays in C57BL/6 mice}

Female C57BL/6 mice (5 weeks old) were obtained from the Medical Experimental Animal Center of Guangdong and maintained in a specific-pathogen-free environment in the animal experimental center of Sun Yat-sen University. All the mice were randomly divided into two groups, six mice in each group. Group 1: mice were injected with Hepa 1-6/LV-IL37 cells; Group 2: mice were injected with Hepa $1-6 / \mathrm{LV}$-NC cells $\left(2 \times 10^{6}\right.$ tumor cells suspended in $100 \mu \mathrm{L}$ of PBS). The length (L) and width (W) of each tumor were measured every 3 days. The tumor volumes were calculated according to the following formula: $\left(\mathrm{L} \times \mathrm{W}^{2}\right) / 2$. Eighteen 
days after tumor cells were inoculated, all mice were sacrificed to harvest, photographed, weighed the tumors. Then, tumors were fixed in formalin overnight, dehydrated, and embedded in paraffin. Tumors were sectioned at a thickness of $2 \mu \mathrm{m}$ to determine IL-37 expression, CD11 $\mathrm{c}^{+}$DCs, $\mathrm{CD} 4^{+} \mathrm{T}$ cells and $\mathrm{CD} 8^{+} \mathrm{T}$ cells infiltration by immunohistochemical analysis. $\mathrm{CD} 11 \mathrm{c}^{+} \mathrm{DCs}, \mathrm{CD} 4^{+} \mathrm{T}$ cells and $\mathrm{CD} 8^{+} \mathrm{T}$ cells were calculated from five fields per section as the mean number of positively stained cells per random highpower microscopic field (HPF, 400× magnification). All procedures of animal experiments in this study were performed according to the guidelines of the laboratory animal ethics committee of Sun Yat-sen University.

\section{Statistical analysis}

All statistical analyses were conducted using SPSS version 19.0 (SPSS Inc., Chicago, IL, USA) or the GraphPad Prism 5 software (GraphPad Software, Inc., La Jolla, CA, USA). Unpaired t-test or the MannWhitney $U$ test was used to compare the differences between two experimental groups. One-way ANOVA was used to compare multiple experimental groups. OS and DFS curves were constructed according to the Kaplan-Meier estimate and were analyzed through the log-rank test. The results are represented as the mean \pm
SD. A two-tailed $P$-value $\leq 0.05$ was considered statistically significant.

\section{Results}

The degree of $\mathrm{CDIa}^{+} \mathrm{DCs}$ infiltration is correlated with IL-37 expression in the tumor tissue of $\mathrm{HCC}$

To explore whether the expression of IL-37 was associated with the infiltration of CD1a ${ }^{+}$DCs in tumor microenvironment of HCC. We examined the infiltration of $\mathrm{CD} \mathrm{a}^{+}$DCs and IL-37 expression in 155 specimens of HCC. Indeed, IL-37 expression was positively correlated with the degree of $\mathrm{CD}^{+} \mathrm{a}^{+}$DCs infiltration (Figure 1A). Comparative analysis further confirmed this trend ( $P=0.038$; Figure 1B). Based on the expression levels of IL-37 and $\mathrm{CD}^{+} \mathrm{a}^{+}$DCs infiltration levels in all 155 patients with $\mathrm{HCC}$, the IL-3 $3{ }^{\text {high }} \mathrm{DC}^{\text {high }}$ patients had the best prognosis (Figure 1C and D).

\section{IL-37 indirectly promotes the migration of DCs by triggering chemokine release from HCC cells}

Then, we explored whether IL-37 has a direct effect on the migration of DCs and its potential mechanism. The
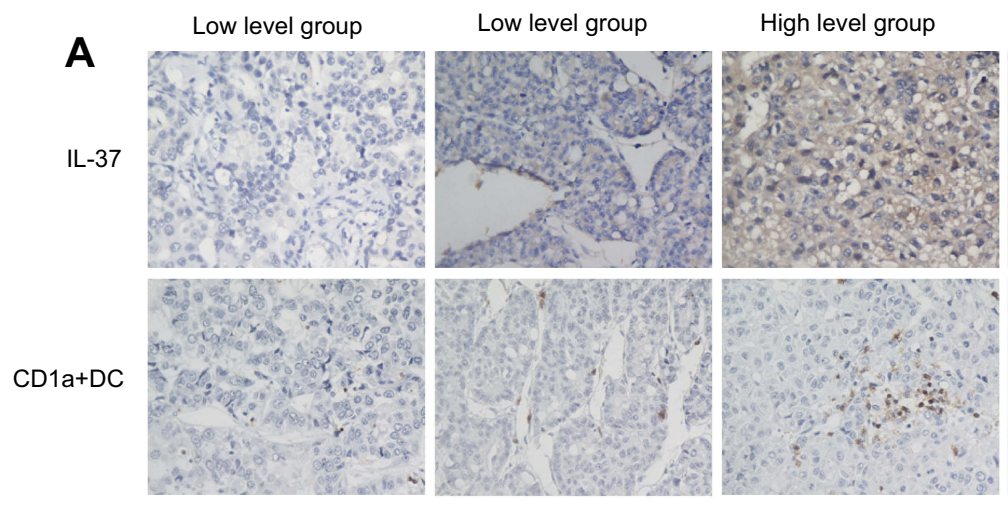

High level group
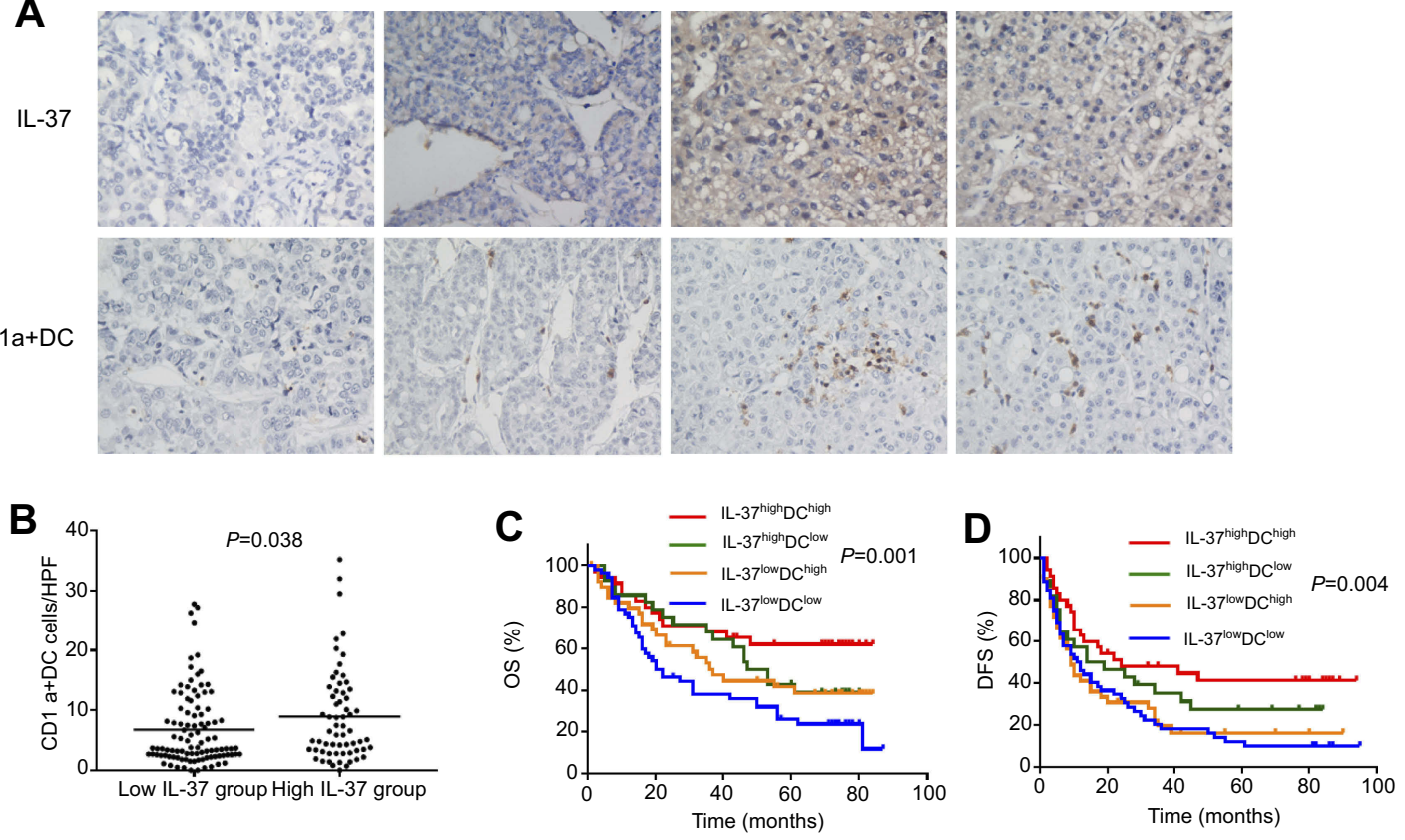

Figure I Relationship between IL-37 expression and CD la ${ }^{+}$DC frequency in the tumor microenvironment. (A) Representative immunohistochemical images of IL-37 and $\mathrm{CDI}$ a in the same primary hepatocellular carcinoma (HCC) tumors (original magnification: $\times 200)$. (B) IL-37 expression positively correlated with the density of CD la ${ }^{+}$DCs in HCC tumors. (C and D) The overall survival (OS) rate and disease-free survival (DFS) rate of Patients. 
lentiviral vector LV-IL37 carrying the gene encoding and control empty vector LV-NC was transduced into the human HCC cell line Hep3B, respectively. Western blotting and ELISA were performed to determine the transfection efficiencies (Figure 2A and $\mathrm{B}$ ). Then, chemotaxis assay was used to test the ability of IL-37-overexpressing HCC cells to recruit DCs in vitro. The result showed that a significantly higher number of DCs recruited by the supernatant of Hep3B/LV-IL37 cells than the supernatant of Hep3B/LV-NC cells, DMEM with recombinant IL-37 (the content of IL-37 in DMEM was similar to that in the supernatant of Hep3B/LV-IL37 cells), or DMEM alone (Figure 2C). However, there were no obvious differences in recruitment by the supernatant of Hep3B/LV-NC cells, DMEM with recombinant IL-37, and DMEM alone. It suggested that IL-37 has no direct effect on the migration of DCs. CCL chemokines were considered to be inducers during the migration of DCs into tumor tissues. ${ }^{20,21}$ To test whether IL-37 had an effect on the production and secretion of CCL chemokines from HCC cells. Therefore, we performed real-time-qPCR to determine the CCL chemokine profiles of Hep3B/LV-NC cells and Hep3B/LV-IL37 cells. Expression levels of mRNAs encoding the chemokines CCL3 and CCL20 were significantly up-regulated in Hep3B/LV-IL37 cells compared to Hep3B/LV-NC cells
(Figure 2D). These results suggested that IL-37 may stimulate chemokine release by tumor cells to recruit DCs into tumor tissue indirectly.

\section{IL-37 indirectly enhances the ability of DCs to induce anti-tumor immune response}

It is well known that the expression of co-stimulatory molecules and MHC like molecules is essential for DCs to induce anti-tumor immune response. Previous studies have demonstrated that decreased expression of co-stimulatory molecules and MHC class molecules present on the surface of DCs can contribute significantly to tumor immune escape. ${ }^{8}$ We examined the effect of IL-37 on the expression of co-stimulatory molecules and MHC molecules on the DC surface. Flow cytometry was used to detect the expression of CD80, CD86, CD40, MHC class I and MHC class II on the surface of DCs. The proportion of CD86, CD40 and MHC class II on the surface of DCs treated with the supernatant of Hep3B/LV-IL37 cells was significantly higher than on the surface of DCs treated with the supernatant of Hep3B/LV-NC cells, DMEM with recombinant IL-37, or DMEM alone. Interestingly, there was no significant difference in the proportion of
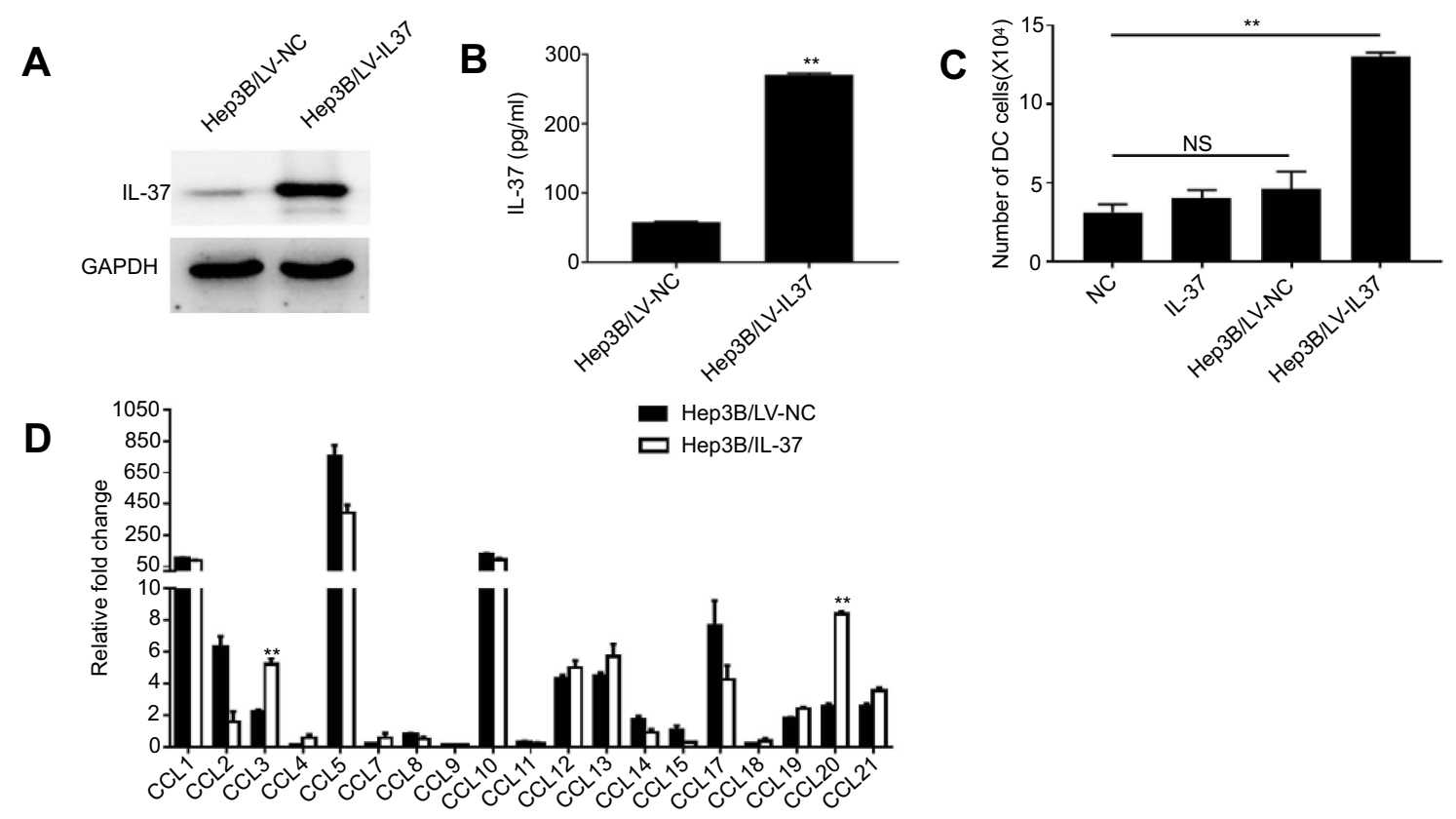

Figure 2 IL-37 recruits dendritic cells (DCs) by stimulating tumor cell-derived CCL chemokine production in hepatocellular carcinoma (HCC). (A) Stable IL-37 expression in Hep3B cells was confirmed by Western blotting. (B) The levels of IL-37 secreted by Hep3B/LV-IL37 cells and Hep3B/LV-NC cells. Data presented are from three separate experiments. (C) Numbers of DCs attracted by DMEM alone, DMEM with recombinant IL-37, supernatant of Hep3B/LV-NC and supernatant of Hep3B/LV-IL37, respectively. Data from three separate experiments are presented. (D) The expression levels of mRNA encoding CCL chemokines in Hep3B/LV-IL37 cells compared to Hep3B/LV-NC cells. Data presented are from three separate experiments. ${ }^{*} P<0.0 \mathrm{I}, \mathrm{NS}$ : not significant. 
CD86 and MHC class II on the surface of DCs treated with the supernatant of Hep3B/LV-NC, DMEM with recombinant IL-37, or DMEM alone. In addition, the proportion of CD40 on the surface of DCs treated with the supernatant of Hep3B/LV-NC or DMEM with recombinant IL-37 were decreased (Figure 3A and B).

IL-12p70 and IFN- $\gamma$, as Th1-type cytokines, enhance the phagocytosis and cytotoxicity of effector cells, including CTLs, NK cells, and macrophages, to promote an antitumor immune response. ${ }^{22,23}$ IL-2, IL-12, and CXCL10 can induce and enhance the CTL anti-tumor immune response. ${ }^{22-26}$ Increased expression and secretion of IL-1, IL- 6 and TNF- $\alpha$ were also essential for the induction of beneficial immune responses by mature DCs. In addition, IFN- $\alpha$ secreted by DC cells played an important role in stimulating its activation and inducing anti-tumor immune response. ${ }^{27}$ However, IL-10 secreted by DC cells inhibited adaptive immune response. ${ }^{28}$ We detected the expression level of chemokines in DCs by real-time-qPCR and found that the DCs treated with the supernatant of Hep3B/LVIL37 cells expressed elevated levels of the mRNA encoding CXCL10 compared with DCs treated with the supernatant of Hep3B/LV-NC, DMEM with recombinant IL-37, or DMEM alone (Figure 3C). The secretion of CXCL10 was determined by ELISA and the same results were obtained (Figure 3D). The levels of IL-2, IL-6, IL-10, IL-12,
IL-12p70, TNF- $\alpha$, IFN- $\alpha$ and IFN- $\gamma$ secreted by DCs were measured by ELISA. Compared with other conditions, the DCs treated with the supernatant of Hep3B/LV-IL37 cells secreted more IL-2, IL-12, IL-12p70, IFN- $\alpha$ and IFN- $\gamma$. (Figure 3D). However, there was no obvious difference between the other conditions. Interestingly, although recombinant IL-37 down-regulated the secretion of IL-6 and TNF$\alpha$ in DCs, no significant differences were found between DCs treated with the supernatant of Hep3B/LV-IL37 and Hep3B/LV-NC cells. And there was no obvious difference in IL-10 secretion among all groups (Figure 3E). These results suggested that IL-37 may indirectly enhance the ability of DCs to induce anti-tumor immune response.

\section{IL-37 indirectly strengthens the cytotoxicity of CTLs induced by DCs}

As the most potent type of major professional APC, the antitumor effect of DCs mainly depends on the action of CTLs, especially $\mathrm{CD} 8^{+} \mathrm{T}_{\text {cells. }}{ }^{8}$ Moreover, IFN- $\gamma$ secreted by CTLs is the most important protective agent against tumor growth. ${ }^{29}$ To investigate whether IL-37 could enhance the anti-tumor effects of CTLs by stimulating DCs to secrete cytokines, we cocultured T lymphocytes with DCs treated with the supernatant of Hep3B/LV-IL37 cells, the supernatant of Hep3B/LV-NC cells, DMEM with recombinant IL-37, or DMEM alone.

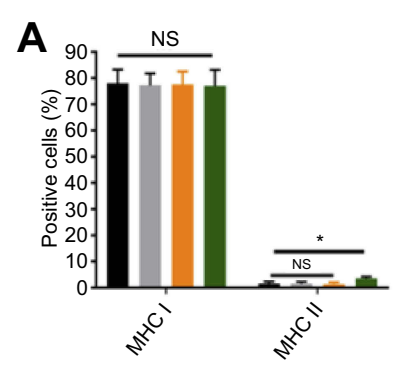

D
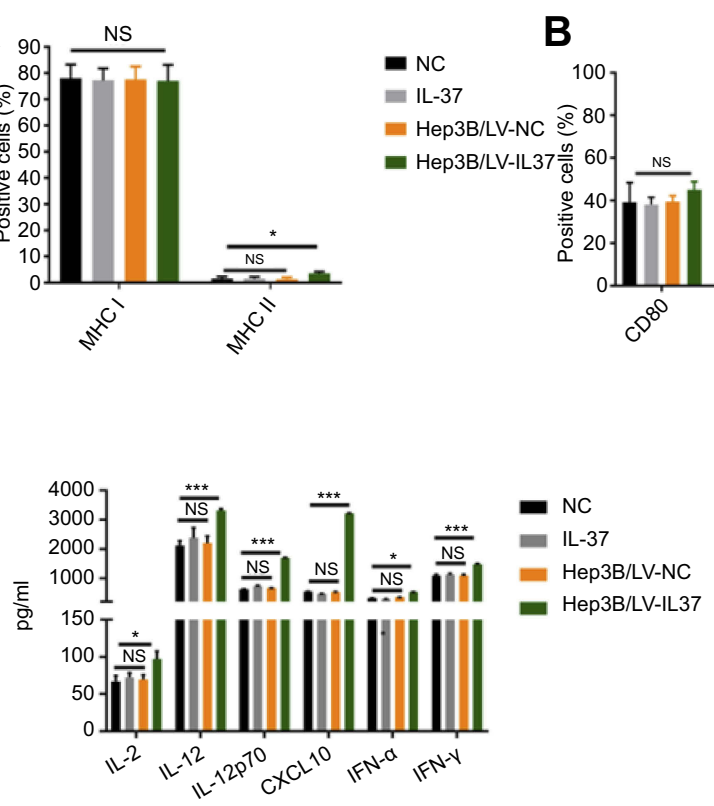

\section{B}

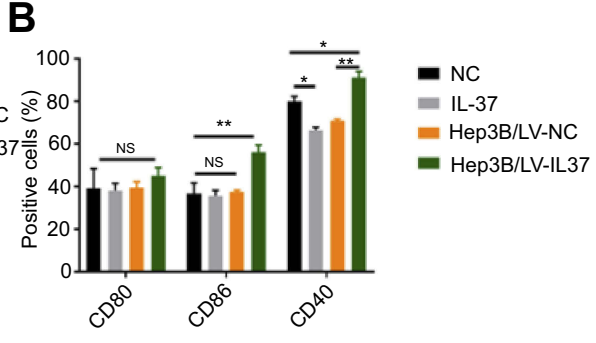

E

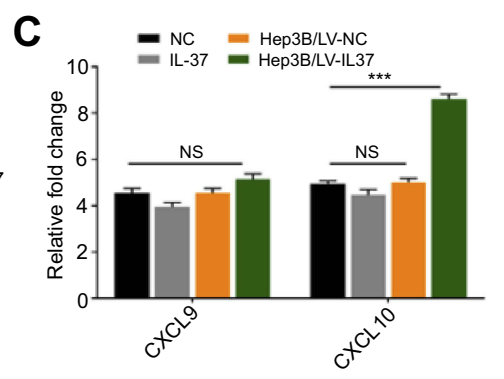

Figure 3 IL-37 indirectly enhances the ability of dendritic cedendritic cells (DCs) to induce anti-tumor immune response. DCs were cultured in DMEM alone, DMEM with recombinant IL-37, supernatant of Hep3B/LV-NC and supernatant of Hep3B/LV-IL37, respectively. (A and B) The expression levels of majorhistocompatibility class (MHC) class I, MHC class II, CD80, CD86 and CD40 on DCs. Data presented are from three separate experiments. (C) The expression levels of the mRNA encoding CXCL9 and CXCLIO in DCs. Data presented are from three separate experiments. (D and E) The levels of IL-2, IL-6, IL-I0, IL-I2, IL-I2p70, CXCLI0, TNF- $\alpha$, interferon- $\boldsymbol{\alpha}$ (IFN- $\boldsymbol{\alpha}$ ) and IFN- $\gamma$ secreted by DCs. Data presented are from three separate experiments. $* P<0.05, * * P<0.01$, $* * * P<0.00 \mathrm{I}$, NS: not significant. 
After 7 days, we tested the proliferative ability of $\mathrm{T}$ lymphocytes through flow cytometry and determined that it was not influenced by the presence of IL-37 (Figure 4A). The level of IFN- $\gamma$ secreted by $\mathrm{T}$ lymphocytes was also examined through flow cytometry. DCs treated with the supernatant of Hep3B/ LV-IL37 cells enhanced IFN- $\gamma$ secretion by T lymphocytes more than DCs treated with the supernatant of Hep3B/LVNC cells, DMEM with recombinant IL-37, or DMEM alone (Figure 4B). More importantly, the proportion of IFN- $\gamma^{+}$T cells and IFN- $\gamma^{+} \mathrm{CD} 8^{+}$T cells had increased significantly (Figure 4C and D). Then, we tested the cytotoxicity of T lymphocytes on Hep3B cells. We found that DCs treated with the supernatant of Hep3B/LV-IL37 cells enhanced the cytotoxicity of T lymphocytes more than DCs treated with the supernatant of Hep3B/LV-NC cells, DMEM with recombinant IL-37, or DMEM alone (Figure 4E). Consequently, IL-37 indirectly strengthens the cytotoxicity of CTLs induced by DCs.

\section{Overexpression IL-37 in HCC cells inhibits tumor growth and increases the recruitment of CDIIc ${ }^{+} \mathrm{DCs}$ in vivo}

To investigate the correlation between the anti-tumor function of IL-37 and the infiltration of DCs in tumor tissues in vivo. We used the lentivirus mentioned untimely to establish stable Hepa 1-6 cell lines (Hepa 1-6/LV-IL37 and Hepa $1-6 / \mathrm{LV}-\mathrm{NC}$ cells). Western blotting and ELISA were performed to determine the transfection efficiencies. Hepa 1-6/LV-IL37 cells $(397.60 \pm 20.67 \mathrm{pg} / \mathrm{mL})$ released a much higher level IL-37 than Hepa $1-6 / \mathrm{LV}-\mathrm{NC}$ cells $(81.55$ $\pm 5.06 \mathrm{pg} / \mathrm{mL}$ ) (Figure $5 \mathrm{~A}$ and $\mathrm{B}$ ). The effect of IL-37 on Hepa $1-6$ cell viability in vitro was confirmed. We found that there was no significant difference in the growth of Hepa 1-6/LV-IL37 and Hepa 1-6/LV-NC cells (Figure 5C). Then, a C57BL/6 mouse model by subcutaneous injection of Hepa 1-6/LV-IL37 and Hepa 1-6/LV-NC cells was established. The result showed that although there is no significant difference in the occurrence time of the tumor, the growth rate of the tumor was obviously slowed down, the tumor volume and weight were reduced in mice injected with Hepa 1-6/LV-IL37 cells $\left(9.39 \pm 2.96 \mathrm{~mm}^{3}, 0.05 \pm 0.01 \mathrm{~g}\right.$, $\mathrm{n}=6)$ compared with Hepa 1-6/LV-NC cells (43.53 \pm 17.37 $\mathrm{mm}^{3}, 0.17 \pm 0.03 \mathrm{~g}, \mathrm{n}=6$ ) (Figure 5D-F). Finally, tumor blocks were surgically removed and collected to evaluate the expression of IL-37 and the infiltration of CD11 ${ }^{+}$DCs, $\mathrm{CD}^{+} \mathrm{T}$ cells and $\mathrm{CD}^{+} \mathrm{T}$ cells via immunohistochemical analysis. Similar to the above findings, the level of $\mathrm{CD}_{11 \mathrm{c}^{+}}$DCs infiltration was significantly higher in the Hepa 1-6/LV-IL37 group (21.5 \pm 1.12 cells/HPF, $n=6)$ than in the Hepa $1-6 / \mathrm{LV}-\mathrm{NC}$ group $(5.83 \pm 0.60$ cells/HPF, $\mathrm{n}=6)$,

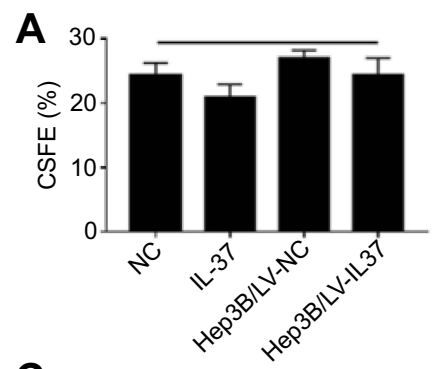

C

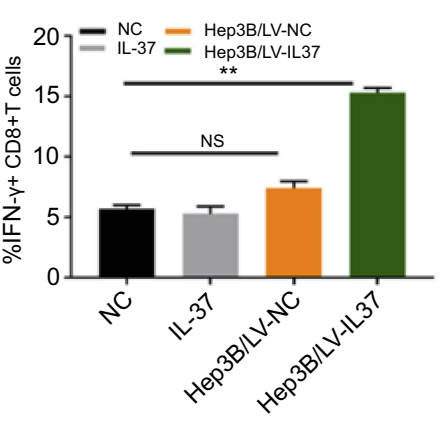

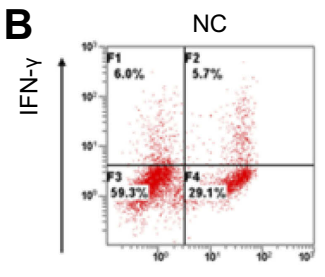
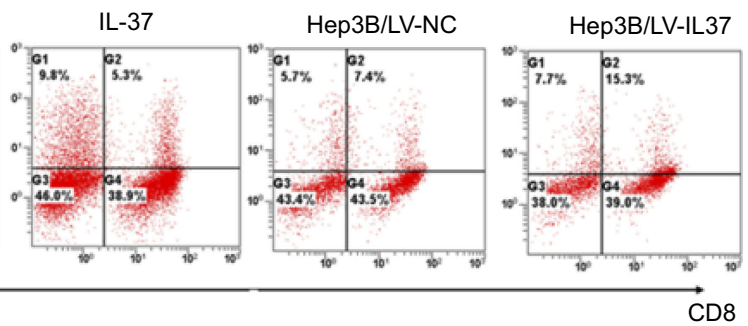

D

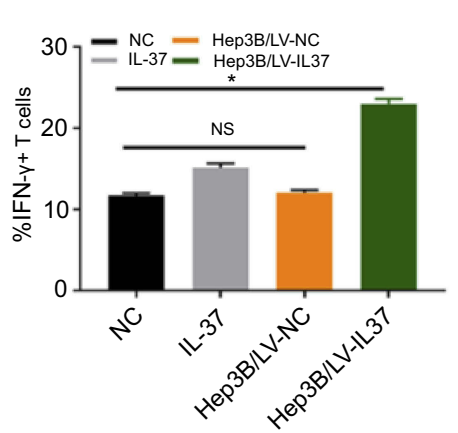

Figure 4 IL-37 indirectly strengthens the cytotoxicity of cytotoxic T lymphocyte (CTL) induced by dendritic cells (DCs). T lymphocytes were treated with DCs cultured in DMEM alone, DMEM with recombinant IL-37, supernatant of Hep3B/LV-NC and supernatant of Hep3B/LV-IL37, respectively. (A) The proliferation of T lymphocytes. (B) The levels of interferon- $\gamma\left(\right.$ IFN- $\gamma$ ) secreted by T lymphocytes. Data presented are from three separate experiments. (C and D) The proportion of IFN- $\gamma^{+}$CD $8^{+} T$ cells and IFN- $\gamma^{+}$ T cells. (E) The cytotoxicity of T lymphocytes. Data presented are from three separate experiments. $* P<0.05$, $* * P<0.01$, NS: not significant. 


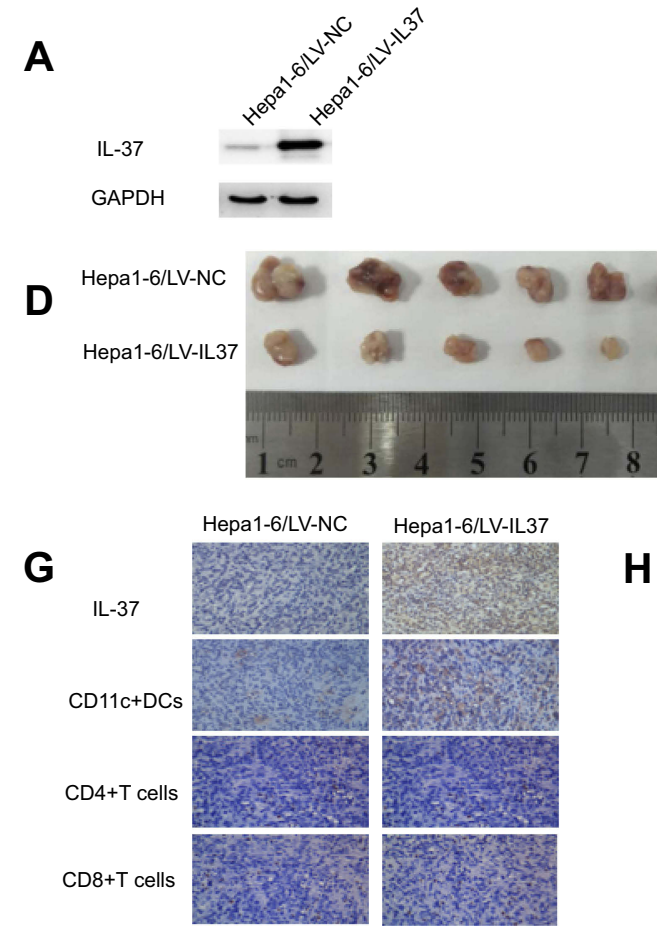

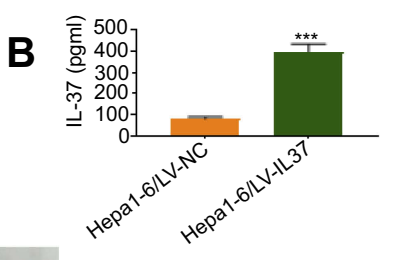

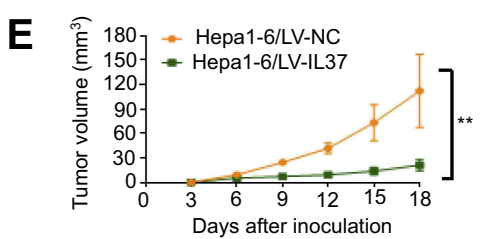

H

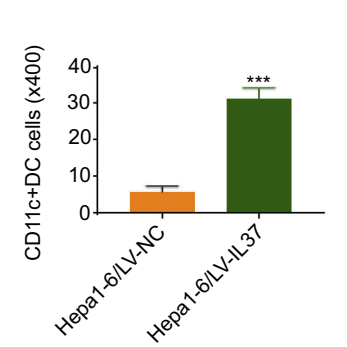

I

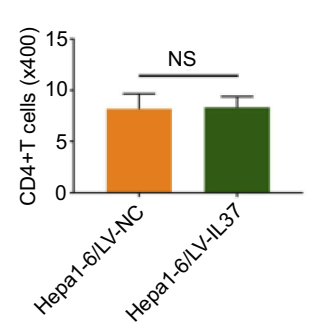

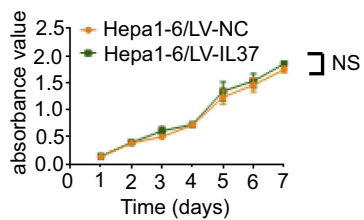
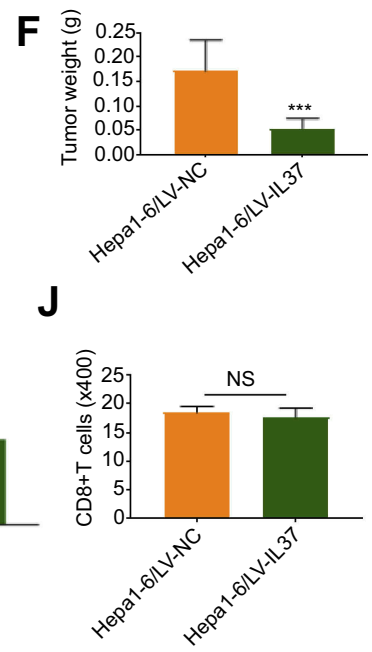

Figure 5 IL-37 indirectly promotes dendritic cell (DC) recruitment and anti-tumor function in vivo. (A) Stable IL-37 expression in Hepa I-6 cells was confirmed by Western blotting. (B) The levels of IL-37 secreted by Hepa I-6/LV-IL37 cells and Hepa I-6/LV-NC cells were detected through ELISA. Data presented are from three separate experiments. (C) MTS assays indicated that overexpression of IL-37 did not significantly affect ex vivo Hepa I-6 cell proliferation. Data presented are from three separate experiments. (D) Photographs of dissected tumors from C57BL/6 mice in the Hepa I-6/LV-IL37 group and Hepa I-6/LV-NC group. (E) Tumor growth curves of Hepa I-6/LV-IL37 group and Hepa I-6/LV-NC group. (F) The final tumor weights of Hepa I-6/LV-IL37 group and Hepa I-6/LV-NC group. (G-J) The expression of IL-37, the density of $C D I I c^{+} D C s, C D 4^{+} T$ cells and $C D 8^{+} T$ cells infiltration in murine tumors (original magnification: $\times 400$ ). Data presented are from six separate experiments. $* * P<0.0$ I, $* * * P<0.00$ I, NS: not significant.

while there was no significant difference for the numbers of intratumoral infiltrating $\mathrm{CD}^{+} \mathrm{T}$ cells and $\mathrm{CD} 8^{+} \mathrm{T}$ cells (Figure 5G-J). These results suggested that IL-37 it might promote the recruitment and activation of DCs in the tumor microenvironment to inhibit tumor progression.

\section{Discussion}

The interplay of a tumor with its microenvironment is crucial to HCC pathogenesis. As an essential modulator of the processes of tumor invasion and metastasis, the interaction between tumor cells and tumor matrix has been extensively studied. The HCC microenvironment consists of hepatoma cells, stromal cells, growth factors, inflammatory cytokines, and extracellular matrix proteins. ${ }^{30}$ Tumor-associated neutrophils, tumor-associated macrophages, and DCs, as immune effector and regulatory cells in the tumor microenvironment, are significant factors in the occurrence and development of malignant tumors. ${ }^{31,32}$ which can be polarized after exposure to the tumor matrix to have either tumorpromoting or anti-tumor effects. These cells are conducive to the proliferation and invasion of cancer cells, angiogenesis of tumor tissues, formation of immunosuppressive microenvironment and pre-metastatic niches in distal tissues.

DCs are essential in adaptive immune responses and antitumor responses. ${ }^{33}$ As a critical participant in tumor immunity and tumor vaccine development, DCs have been widely concerned. ${ }^{34}$ Accumulating evidence suggests that the tumor microenvironment prevents DCs maturation or polarizes DCs differentiation; then, the ability of DCs to take up and deliver antigen to lymphocyte cells is reduced or even disappeared. ${ }^{35}$ However, the function of DCs in the tumor microenvironment can be regulated to restore their role in the anti-tumor immune response. IL-12 can reverse the damage of allogeneic T cell stimulation by DCs. ${ }^{36}$ Expression of co-stimulatory molecules and the ability of inducing tumor-specific cytotoxic cells were enhanced of DCs overexpressing IL-12. ${ }^{37}$ IL-24 exerts antitumor effects by significantly increasing the expression of costimulatory molecules and $\mathrm{MHC}$ molecules on the surface of DCs and improving the antigen-presenting ability of DCs. ${ }^{38}$ Cao et al found that DCs transfected with IL-18 can induce stronger therapeutic anti-tumor immunity. ${ }^{39}$ Moreover, it has been reported that IL-37 can mediate the combined anti-tumor 
effects of IL-12 and IL-18. ${ }^{14}$ These studies indicate that some cytokines can exert an anti-tumor effect by regulating DCs function.

IL-37, a cytokine of the IL-1 family. Studies of many types of tumors showed that IL-37 has a tumor suppressive effect, colon cancer, cervical cancer, and prostate cancer included. $^{15,17,18}$ Gao et al confirmed that IL-37 could significantly inhibit the growth of sarcoma; moreover, the antitumor immunoreaction of IL-37 is dependent on T cells and B cells, but not NK cells. ${ }^{14}$ Previously, we demonstrated that IL-37 also showed a tumor suppressive effect in HCC. HCC cells overexpressing IL-37 might recruit NK cells into tumor stroma and induce an enhanced anti-tumor response. ${ }^{19}$

In this study, the expression level of IL-37 was positively associated with the degree of $\mathrm{CD} \mathrm{a}^{+} \mathrm{DCs}$ infiltration in $155 \mathrm{HCC}$ patients, and the IL- $37^{\text {high }} \mathrm{DC}^{\text {high }}$ patients were found to have the best prognoses. The results of chemotaxis assays and mouse experiments were consistent with tissue specimen from patient with HCC. In a previous study, Cai et al also found that the infiltration of lymphocytes and DCs in tumor tissues of $\mathrm{HCC}$, as an independent prognostic factor, was closely related to postoperative prognosis. ${ }^{40}$ In addition, we found that IL-37 has no direct effect on the migration of DCs. It stimulated chemokine release by tumor cells to recruit DCs into tumor tissue indirectly. Interestingly, we found that recombinant IL-37 decreases the expression of CD40 on DCs and down-regulated the secretion of IL- 6 and TNF- $\alpha$ in DCs. While HCC cells expressing IL-37 increase the expression of CD86, CD40 and MHC class II on DCs and up-regulated the secretion of IL-2, IL-12, IL-12p70, IFN- $\alpha$ and IFN- $\gamma$. This is not entirely consistent with previous studies. Luo et al suggested that IL-37 treatment decreases the expression of the co-stimulating molecules on DCs and down-regulates the expression of the cytokines IL-1, IL-6 and TNF- $\alpha$ at mRNA and protein levels; in addition, IL-37 treatment reduces the CXCR1 expression and migration ability of DCs. ${ }^{41}$ It may be due to the fact that the tumor microenvironment is inconsistent with the acute inflammatory environment, and IL-37 could indirectly regulate DC function in other ways. We also found that IL-37 could enhance the ability of DCs to induce the anti-tumor effects of CTLs by stimulating HCC cells. In mouse model, the level of $\mathrm{CD}_{11 \mathrm{c}^{+}}$DCs infiltration was significantly higher in the Hepa 1-6/LV-IL37 group than in the Hepa 1-6/LV-NC group, while there was no significant difference for the numbers of intratumoral infiltrating $\mathrm{CD}^{+}{ }^{+} \mathrm{T}$ cells and $\mathrm{CD}^{+} \mathrm{T}$ cells. All the above results indicate that IL-37 may be a potent activator for the maturation of DCs in HCC. IL-
37 could induce DCs to secrete cytokines and then enhance the anti-tumor effects of $\mathrm{T}$ lymphocytes by stimulating HCC cells. However, the potential molecular mechanisms of IL-37 regulating chemokines and the function of DCs still need to be further explored.

\section{Conclusion}

In summary, our study demonstrated that IL-37 expression was positively correlated with the degree of DCs infiltration in primary HCC tumors; moreover, both high expression of IL-37 and high infiltration by CD1a ${ }^{+}$DCs were associated with better prognosis of patients with HCC. An increase of IL-37 expression in HCC cells intensifies their ability to recruit DCs into tumor tissues. IL-37 subsequently promote maturation of DCs and induce secretion of cytokines to augment the anti-tumor effects of CTLs by stimulating HCC cells. Thus, the positive regulation of IL-37 on DCmediated anti-tumor immune responses may contribute to develop more effective immunotherapies for HCC.

\section{Acknowledgments}

This work was supported by grants from the National Key Research and Development Program of China (No. 2018YFC1313400), the National Natural Science Foundation of China (No.81402560; 81572865; 81472387), Guangdong Esophageal Cancer Institute Science and Technology Program (No. Q201802) and the Guangdong Province Science and Technology Plan Project (No. 2017A020215029; 2014A020212584; 2013B021800063).

\section{Author contributions}

All authors contributed to data analysis, drafting or revising the article, gave final approval of the version to be published, and agree to be accountable for all aspects of the work.

\section{Disclosure}

The authors declare that they have no competing interests in this work.

\section{References}

1. Laursen L. A preventable cancer. Nature. 2014;516(7529):S2-S3. doi:10.1038/516S2a

2. Tabrizian P, Roayaie S, Schwartz ME. Current management of hepatocellular carcinoma. World J Gastroenterol. 2014;20(30):1022310237. doi:10.3748/wjg.v20.i30.10223

3. Erstad DJ, Tanabe KK. Hepatocellular carcinoma: early-stage management challenges. J Hepatocell Carcinoma. 2017;4:81-92. doi:10.2147/ JHC.S107370 
4. Galun D, Srdic-Rajic T, Bogdanovic A, Loncar Z, Zuvela M. Targeted therapy and personalized medicine in hepatocellular carcinoma: drug resistance, mechanisms, and treatment strategies. $J$ Hepatocell Carcinoma. 2017;4:93-103. doi:10.2147/JHC. S106529

5. Lu LC, Hsu CH, Hsu C, Cheng AL. Tumor heterogeneity in hepatocellular carcinoma: facing the challenges. Liver Cancer. 2016;5 (2):128-138. doi:10.1159/000367754

6. Hansen M, Andersen MH. The role of dendritic cells in cancer. Semin Immunopathol. 2017;39(3):307-316. doi:10.1007/s00281-016-0592-y

7. Santos PM, Butterfield LH. Dendritic cell-based cancer vaccines. $J$ Immunol. 2018;200(2):443-449. doi:10.4049/jimmunol.1701024

8. Harimoto H, Shimizu M, Nakagawa Y, et al. Inactivation of tumorspecific CD8(+) CTLs by tumor-infiltrating tolerogenic dendritic cells. Immunol Cell Biol. 2013;91(9):545-555. doi:10.1038/icb.2013.38

9. Wu SD, Ma YS, Fang Y, Liu LL, Fu D, Shen XZ. Role of the microenvironment in hepatocellular carcinoma development and progression. Cancer Treat Rev. 2012;38(3):218-225. doi:10.1016/j. ctrv.2011.06.010

10. Han Y, Chen Z, Yang Y, et al. Human CD14+ CTLA-4+ regulatory dendritic cells suppress T-cell response by cytotoxic T-lymphocyte antigen-4-dependent IL-10 and indoleamine-2,3-dioxygenase production in hepatocellular carcinoma. Hepatology. 2014;59(2):567-579. doi:10.1002/hep. 26694

11. Kakumu S, Ito S, Ishikawa T, et al. Decreased function of peripheral blood dendritic cells in patients with hepatocellular carcinoma with hepatitis B and C virus infection. J Gastroenterol Hepatol. 2000;15 (4):431-436.

12. Chen S, Akbar SM, Tanimoto K, et al. Absence of CD83-positive mature and activated dendritic cells at cancer nodules from patients with hepatocellular carcinoma: relevance to hepatocarcinogenesis. Cancer Lett. 2000;148(1):49-57.

13. Wu B, Meng K, Ji Q, et al. Interleukin-37 ameliorates myocardial ischaemia/reperfusion injury in mice. Clin Exp Immunol. 2014;176 (3):438-451. doi:10.1111/cei.12284

14. Gao W, Kumar S, Lotze MT, Hanning C, Robbins PD, Gambotto A. Innate immunity mediated by the cytokine IL-1 homologue 4 (IL1H4/IL-1F7) induces IL-12-dependent adaptive and profound antitumor immunity. J Immunol. 2003;170(1):107-113. doi:10.4049/ jimmunol.170.1.107

15. Ge G, Wang A, Yang J, et al. Interleukin-37 suppresses tumor growth through inhibition of angiogenesis in non-small cell lung cancer. $J$ Exp Clin Cancer Res. 2016;35:13. doi:10.1186/s13046-016-0444-6

16. Yan X, Zhao J, Zhang R. Interleukin-37 mediates the antitumor activity in colon cancer through beta-catenin suppression. Oncotarget. 2017;8(30):49064-49075. doi:10.18632/oncotarget.170 93

17. Wang S, An W, Yao Y, et al. Interleukin 37 expression inhibits STAT3 to suppress the proliferation and invasion of human cervica cancer cells. J Cancer. 2015;6(10):962-969. doi:10.7150/jca.12266

18. Wang WQ, Zhao D, Zhou YS, et al. Transfer of the IL-37b gene elicits anti-tumor responses in mice bearing 4T1 breast cancer. Acta Pharmacol Sin. 2015;36(4):528-534. doi:10.1038/aps.2015.3

19. Zhao JJ, Pan QZ, Pan K, et al. Interleukin-37 mediates the antitumor activity in hepatocellular carcinoma: role for CD57+ NK cells. Sci Rep. 2014;4:5177. doi:10.1038/srep05177

20. He S, Wang L, Wu Y, Li D, Zhang Y. CCL3 and CCL20-recruited dendritic cells modified by melanoma antigen gene-1 induce antitumor immunity against gastric cancer ex vivo and in vivo. $J \operatorname{Exp}$ Clin Cancer Res. 2010;29:37. doi:10.1186/1756-9966-29-17

21. Holcmann M, Drobits B, Sibilia M. How imiquimod licenses plasmacytoid dendritic cells to kill tumors. Oncoimmunology. 2012;1 (9):1661-1663. doi:10.4161/onci.22033

22. Kalinski P. Dendritic cells in immunotherapy of established cancer: roles of signals 1, 2, 3 and 4. Curr Opin Investig Drugs. 2009;10 (6):526-535.
23. Langenkamp A, Messi M, Lanzavecchia A, Sallusto F. Kinetics of dendritic cell activation: impact on priming of TH1, TH2 and nonpolarized T cells. Nat Immunol. 2000;1(4):311-316. doi:10.1038/ 79758

24. Berk E, Kalinski P. Lymphocyte-polarized DC1s: effective inducers of tumor-specific CTLs. Oncoimmunology. 2012;1(8):1443-1444. doi:10.4161/onci.21295

25. de Jong EC, Smits HH, Kapsenberg ML. Dendritic cell-mediated T cell polarization. Springer Semin Immunopathol. 2005;26(3):289307. doi:10.1007/s00281-004-0167-1

26. Salcedo R, Hixon JA, Stauffer JK, et al. Immunologic and therapeutic synergy of IL-27 and IL-2: enhancement of T cell sensitization, tumor-specific CTL reactivity and complete regression of disseminated neuroblastoma metastases in the liver and bone marrow. $J$ Immunol. 2009;182(7):4328-4338. doi:10.4049/jimmunol.0800471

27. Medrano RFV, Hunger A, Mendonca SA, Barbuto JAM, Strauss BE. Immunomodulatory and antitumor effects of type I interferons and their application in cancer therapy. Oncotarget. 2017;8(41):71249 71284. doi:10.18632/oncotarget. 19531

28. Ma Y, Shurin GV, Gutkin DW, Shurin MR. Tumor associated regulatory dendritic cells. Semin Cancer Biol. 2012;22(4):298-306. doi:10.1016/j.semcancer.2012.02.010

29. McCormack R, de Armas L, Shiratsuchi M, Podack ER. Killing machines: three pore-forming proteins of the immune system. Immunol Res. 2013;57(1-3):268-278. doi:10.1007/s12026-013-8469-9

30. Yang JD, Nakamura I, Roberts LR. The tumor microenvironment in hepatocellular carcinoma: current status and therapeutic targets. Semin Cancer Biol. 2011;21(1):35-43. doi:10.1016/j.semcancer.2010.10.007

31. Fridlender ZG, Sun J, Kim S, et al. Polarization of tumor-associated neutrophil phenotype by TGF-beta: "N1" versus "N2" TAN. Cancer Cell. 2009;16(3):183-194. doi:10.1016/j.ccr.2009.06.017

32. Tran Janco JM, Lamichhane P, Karyampudi L, Knutson KL. Tumorinfiltrating dendritic cells in cancer pathogenesis. J Immunol. 2015;194(7):2985-2991. doi:10.4049/jimmunol.1403134

33. Strioga M, Schijns V, Powell DJ Jr., Pasukoniene V, Dobrovolskiene N, Michalek J. Dendritic cells and their role in tumor immunosurveillance. Innate Immun. 2013;19(1):98-111. doi:10.1177/17534259 12449549

34. Steinman RM, Banchereau J. Taking dendritic cells into medicine. Nature. 2007;449(7161):419-426. doi:10.1038/nature06175

35. Steinman RM, Hawiger D, Nussenzweig MC. Tolerogenic dendritic cells. Annu Rev Immunol. 2003;21:685-711. doi:10.1146/annurev. immunol.21.120601.141040

36. Ormandy LA, Farber A, Cantz T, et al. Direct ex vivo analysis of dendritic cells in patients with hepatocellular carcinoma. World $J$ Gastroenterol. 2006;12(20):3275-3282. doi:10.3748/wjg.v12.i20.3275

37. Vogt A, Sievers E, Lukacs-Kornek V, et al. Improving immunotherapy of hepatocellular carcinoma (HCC) using dendritic cells (DC) engineered to express IL-12 in vivo. Liver Int. 2014;34(3):447-461. doi:10.1111/liv. 12284

38. Yu X, Xia W, Zhang T, et al. Enhanced cytotoxicity of IL-24 genemodified dendritic cells co-cultured with cytokine-induced killer cells to hepatocellular carcinoma cells. Int J Hematol. 2010;92(2):276282. doi:10.1007/s12185-010-0654-1

39. Cao DY, Yang JY, Dou KF, Ma LY, Teng ZH. alpha-fetoprotein and interleukin-18 gene-modified dendritic cells effectively stimulate specific type-1 CD4- and CD8-mediated T-Cell response from hepatocellular carcinoma patients in Vitro. Hum Immunol. 2007;68(5):334341. doi:10.1016/j.humimm.2007.01.008

40. Cai XY, Gao Q, Qiu SJ, et al. Dendritic cell infiltration and prognosis of human hepatocellular carcinoma. J Cancer Res Clin Oncol. 2006;132(5):293-301. doi:10.1007/s00432-006-0075-y

41. Luo Y, Cai X, Liu S, et al. Suppression of antigen-specific adaptive immunity by IL-37 via induction of tolerogenic dendritic cells. Proc Natl Acad Sci U S A. 2014;111(42):15178-15183. doi:10.1073/ pnas. 1416714111 


\section{Supplementary material}

Table SI Primers for real-time quantitative polymerase chain reaction analysis

\begin{tabular}{|c|c|c|}
\hline Genes & Sense & Antisense \\
\hline CCLI & CATTTGCGGAGCAAGAGATT & TGCCTCAGCATTTTTTCTGTG \\
\hline $\mathrm{CCL} 2$ & GCCTCCAGCATGAAAGTCTC & CAGATCTCCTTGGCCACAAT \\
\hline $\mathrm{CCL} 3$ & TGCTCAGAATCATGCAGGTC & GGGAGGTGTAGCTGAAGCAG \\
\hline CCL4 & GAGTTCTGCAGCCTCACCTC & СTCACTGGGATCAGCACAGA \\
\hline CCL5 & AGCTACTCGGGAGGCTAAGG & GAGGCATGCTGACTTCCTTC \\
\hline CCL7 & ATGAAAGCCTCTGCAGCACT & GGACAGTGGCTACTGGTGGT \\
\hline CCL8 & TCACCTGCTGCTTTAACGTG & GGGAGGTTGGGGAAAATAAA \\
\hline CCL9 & TGTGCCAACTCTCTGCATTC & GACCTCAGCTTAGGGCAGTG \\
\hline CCLIO & TTTGAAACGAACAGCGAGTG & TGTGTCCAGCTTCAGCATTC \\
\hline CCLII & CAGAGAGGCTGAGACCAACC & CACAGCTTTCTGGGGACATT \\
\hline CCLI2 & GCCTTCTGTTCCTTGGTGTC & TGTACCTCTGGACCCACTCC \\
\hline CCLI3 & ATCTCCTTGCAGAGGCTGAA & AGAAGAGGAGGCCAGAGGAG \\
\hline CCLI4 & ССАТТСССТТСТТССТССТС & TTGTCACTGGGGTTGGTACA \\
\hline CCLI5 & GGACTTCCTGGATCCTCCTC & AGCAGTCAGCAGCAAAGTGA \\
\hline CCLI7 & АCTGTCTCCCGGGACTACCT & TTTAATCTGGGCCCTTTGTG \\
\hline CCLI8 & ТССССТТТСССТТСААСТСТ & GTGCTGAGCAAAACCATTCA \\
\hline CCLI9 & GACCTCAGCCAAGATGAAGC & GGTCCTTCCTTCTGGTCCTC \\
\hline CCL20 & GTGGCTTTTCTGGAATGGAA & CAAGTCCAGTGAGGCACAAA \\
\hline CCL2I & GCCTTGCCACACTCTTTCTC & CAAGGAAGAGGTGGGGTGTA \\
\hline CXCL9 & TCTGATTGGAGTGCAAGGAACC & GGTCTTTCAAGGATTGTAGGTGGA \\
\hline CXCLIO & GCCTCTCCCATCACTTCCСTAC & GAAGCAGGGTCAGAACATCCAC \\
\hline
\end{tabular}

\section{Publish your work in this journal}

Cancer Management and Research is an international, peer-reviewed open access journal focusing on cancer research and the optimal use of preventative and integrated treatment interventions to achieve improved outcomes, enhanced survival and quality of life for the cancer patient.
The manuscript management system is completely online and includes a very quick and fair peer-review system, which is all easy to use. Visit http://www.dovepress.com/testimonials.php to read real quotes from published authors. 